\title{
Reducción de la viscosidad a través de un inductor térmico
}

\author{
Viscosity reduction through a thermal inductor
}

\section{Eglys Rodríguez}

eglysdvrg@gmail.com

Código ORCID: 0000-0002-3076-0719

Universidad del Zulia, Núcleo Costa Oriental del Lago, Venezuela

Resumen

Se identificaron los elementos de la gestión de mantenimiento presentes en las unidades de bombeo mecánico convencionales. El estudio fue descriptivo, con diseño no experimental, transeccional, y de campo. La población quedó conformada por los gerentes y supervisores de las unidades de bombeo mecánico convencionales de Petróleos de Venezuela S. A. Para la recolección de datos se aplicó la encuesta, a través de un cuestionario contentivo de 25 ítems, utilizando una escala dicotómica. La validación se realizó mediante el juicio de expertos. La confiabilidad se calculó a través del coeficiente Kuder y Richardson, donde se obtuvo 0,93. El análisis de los datos se realizó mediante las frecuencias registradas. Se concluye del análisis realizado alta presencia en la gestión de mantenimiento analizada, señalando como elementos de la gestión la planificación, programación, ejecución, control y evaluación, es decir, el conjunto de acciones pertinentes para su cumplimiento.

Palabras clave: Control, ejecución, elementos de la gestión de mantenimiento, evaluación, planificación, programación

\section{Abstract}

Theelements

of

maintenancemanagementpresent

in conventionalmechanicalpumpingunitswereidentified. Thestudywasdescriptive, with a nonexperimental, transectional, and fielddesign. Thepopulationwasmade up of the managers and supervisors of theconventionalmechanicalpumpingunits of Petróleos de Venezuela $S$. A. Forthe data collection, thesurveywasappliedthrough a questionnairecontaining 25 items, using a dichotomousscale.

Validationwasperformedthroughexpertjudgment. ReliabilitywascalculatedusingtheKuder and Richardson coefficient, where 0.93 wasobtained. Data analysiswasperformedusingtherecordedfrequencies.

Itisconcludedfromtheanalysiscarriedouthighpresence in themaintenancemanagementanalyzed, indicating as planningelements, planning, organization, programming, execution, control and evaluation, thatis to say, the set of pertinentactionsforitsfulfillment.

Keywords: Control, execution, elements of maintenancemanagement, evaluation, planning, programming 
INTRODUCCIÓN

En la producción de crudos pesados una de las maneras de estimular los pozos con altos valores de viscosidad es mediante la utilización de la inyección alternada de vapor (IAV), la cual está orientada a la disminución de la viscosidad al incrementar la temperatura del fluido para obtener una mayor tasa de producción en los pozos.

A este respecto, la viscosidad es una característica muy importante que controla el flujo de petróleo a través del medio poroso y de las tuberías. Generalmente se define como la resistencia interna que ofrece el petróleo para moverse. Esta propiedad depende fuertemente de la temperatura del yacimiento, la presión, la gravedad del petróleo, la gravedad y solubilidad del gas (Petróleos de Venezuela, 2013).

Dentro de este contexto científico, algunas técnicas ampliamente empleadas y estudiadas para la recuperación mejorada de crudos muy viscosos son la inyección de vapor o de agua caliente, la inyección de fluidos miscibles y la combustión en sitio. Estas técnicas se basan en la introducción de calor al yacimiento con el objetivo de reducir la viscosidad del crudo allí presente, así como la reducción de las fuerzas capilares (Salageret al, 2005).

Sin embargo, estas técnicas presentan inconvenientes cuando la inyectividad del yacimiento es pobre, existe la presencia de acuíferos, las arenas son muy delgadas, los yacimientos presentan altas presiones 0 cuando se encuentran en áreas de difícil acceso. Otros problemas presentados son la baja eficiencia en el barrido de crudo, la canalización del fluido inyectado y las emisiones de gases contaminantes, resultado de la inyección.

Mientras que, existe un cierto número de pozos donde el crudo está en un rango de 400 hasta los 200.0000 centipoise (Cps), catalogado como crudo de alta viscosidad, condición que ocasiona una alta fricción cabilla-fluido en el sistema de levantamiento artificial de bombeo mecánico (BM) y alto torque en el bombeo de cavidad progresiva (BCP), lo cual genera una baja eficiencia en el sistema de levantamiento y ocasiona a la vez una producción diferida, alto costo por operación y mantenimiento mayor.

Para tal efecto, se planteó como reto tecnológico la aplicación de mecanismos de recuperación mejorada de hidrocarburos, en lugar de mantener el mismo esquema de explotación usando procesos convencionales de producción primaria y secundaria. Específicamente, los alcances de aplicación están orientados al método de calentamiento eléctrico usando el inductor térmico NOKALT 4500, cuyos componentes se muestran en la Figura 1. para optimizar el activo de la empresa y generar un incremento del potencial de producción a corto plazo, garantizándose de esta manera la operatividad eficiente y sostenida del Campo. 
De acuerdo con las ideas expuestas, NOKALT 4500, que permita incrementar surge la idea de establecer una la temperatura del crudo en fondo del metodología de selección de pozos con pozo, para reducir su viscosidad y problemas de alta fricción cabilla-fluido mejorar su movilidad, y logrando a la (BM) y de alto torque (BCP), que vez, de esta manera, optimizar el cumplan con los requisitos mínimos para sistema de levantamiento artificial para la instalación del inductor térmico recuperar la producción diferida.

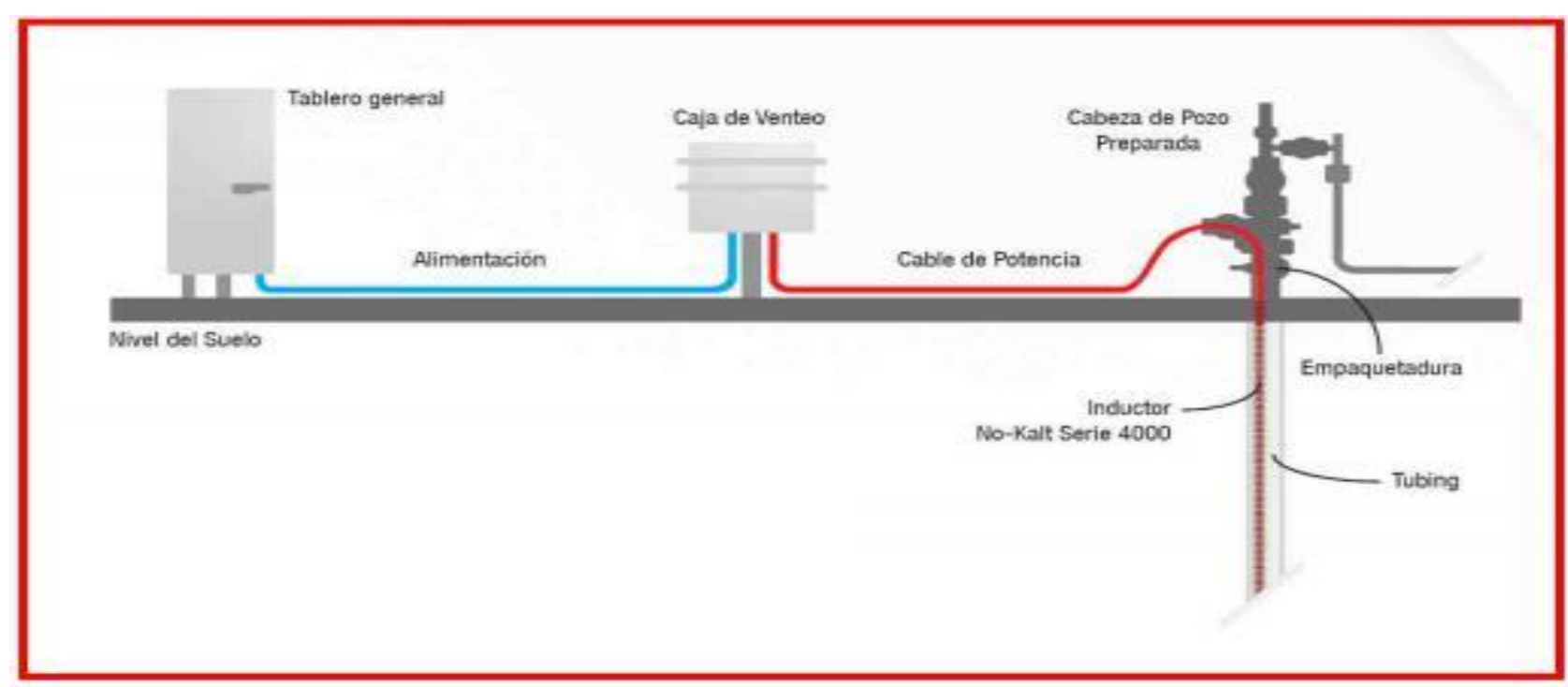

Figura 1. Componentes de superficie del Inductor Térmico NOKALT 4500. Fuente: PDVSA (2015) 
Por ello se planteó como objetivo determinar la factibilidad técnica para el uso de un inductor térmico para reducir la viscosidad en pozos de crudo, como alternativa de solución para mejorar la tasa de producción de los pozos ubicados en áreas donde se imposibilita el proceso de inyección alternada de vapor (IAV).

\section{MÉTODO}

La investigación se ubicó como un modelo, en un área particular del conocimiento a partir de un diagnóstico preciso de las necesidades del momento; los procesos explicativos o generadores involucrados y las tendencias futuras, en lo que se conoce como proyectiva (Hurtado, 2006). Bajo un diseño documental, donde la recolección de los datos de producción y la información necesaria sobre los eventos ocurridos en los pozos se hizo directamente de archivos, informes, expedientes, base de datos, registros, sin manipular variable alguna ni realizar ningún cambio con el propósito de interpretarlos, entender su naturaleza y crear procesos de simulación exitosos, con una incertidumbre muy baja.

En un contexto de estudio, seleccionados a través de una matriz escalonada, que cumplieron con premisas o condiciones expuestas en el procedimiento metodológico y de los cuales fueron escogidos diez (10) pozos para un estudio de simulación, reseñados a través de PDVSA y la empresa Global Technologies, las cuales instalaron dos (2) equipos No-Kalt $\circledast$ en Occidente - División Costa Oriental del
Lago, para realizar pruebas in situ de la tecnología

De manera que, para la recolección de la data sobre viscosidad se utilizó la correlación de Beggs, utilizando la viscosidad del petróleo muerto (libre de gas) en función de la gravedad API y varios valores de temperatura. Con el fin de obtener una expresión que involucre los grados ${ }^{\circ} \mathrm{API}$ del crudo, se partió de las correlaciones existentes en la teoría, cuyos coeficientes fueron adaptados a las condiciones del campo, mediante usos de artificios matemáticos que plantean sistemas de ecuaciones. Y a partir del programa computacional centinela, se obtuvo información referente a pruebas de producción de los pozos que incluyen Grados API, barriles totales producidos de petróleo, agua y gas, corte de agua, RGP, entre otros.

Para la evaluación del comportamiento de producción de los pozos se utilizó el programa inflow performance relationship elaborado por PDVSA, donde se procedió a construir una curva de afluencia, para construir dicha curva se utilizaron los siguientes datos: Presión promedio del reservorio, presión dinámica del test y el caudal del test, los cuales fueron recolectados por fuentes bibliográficas. Una vez obtenidas las curvas se procedió a evaluar el cambio de producción que se produjo desde el momento que fue instalada la tecnología de inductor térmico NOKALT 4500.

Posterior a ello, se utilizó una matriz escalonada con la finalidad de establecer una evaluación de variables 
que poseen mayor influencia al momento de seleccionar los pozos para instalar la tecnología de inductor térmico NOKALT 4500, la misma permitió evaluar cómo es el comportamiento de cada variable respecto a otra, la cual se realizó mediante la matriz de evaluación de pozos. La Tabla 1 muestrala matriz escalonada, la evaluación de las variables con respecto a otra, donde cada valor total de las variables fue multiplicado por el valor adjudicado a cada variable de cada uno de los pozos previamente seleccionado en la matriz de evaluación.

Tabla 1. Matriz escalonada

\begin{tabular}{|c|c|c|c|c|c|c|c|c|c|c|c|c|c|}
\hline Variables & 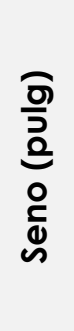 & $\begin{array}{l}\text { 츨 } \\
\text { 몸 }\end{array}$ & 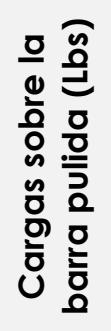 & 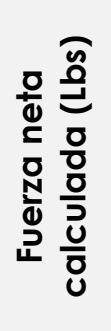 & 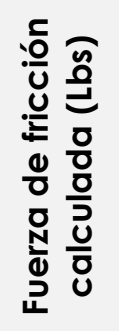 & 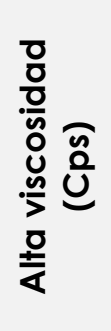 & 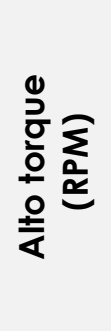 & 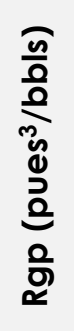 & $\frac{\pi}{\grave{\alpha}}$ & 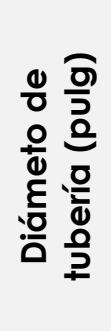 & 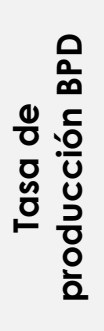 & 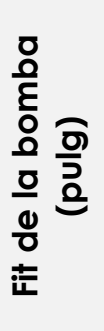 & 홍 \\
\hline Seno (pulg) & 0 & 7 & 9 & 8 & 9 & 10 & 9 & 4 & 6 & 6 & 7 & 8 & 83 \\
\hline THP (psi) & 7 & 0 & 6 & 5 & 6 & 9 & 10 & 4 & 8 & 6 & 9 & 6 & 76 \\
\hline $\begin{array}{l}\text { Cargas sobre la } \\
\text { barra pulida } \\
\text { (Lbs) }\end{array}$ & 9 & 6 & 0 & 7 & 6 & 8 & 8 & 4 & 5 & 6 & 5 & 7 & 71 \\
\hline $\begin{array}{l}\text { Fuerza neta } \\
\text { calculada (Lbs) }\end{array}$ & 8 & 5 & 7 & 0 & 6 & 7 & 8 & 2 & 3 & 6 & 5 & 7 & 64 \\
\hline $\begin{array}{l}\text { Fuerza de } \\
\text { fricción } \\
\text { calculada (Lbs) }\end{array}$ & 9 & 6 & 6 & 6 & 0 & 8 & 8 & 4 & 5 & 6 & 6 & 6 & 70 \\
\hline
\end{tabular}




\begin{tabular}{|c|c|c|c|c|c|c|c|c|c|c|c|c|c|}
\hline Variables & 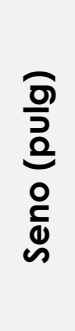 & 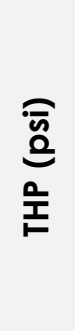 & 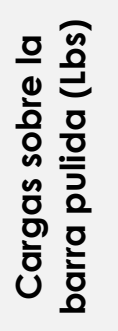 & 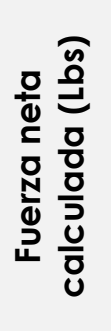 & 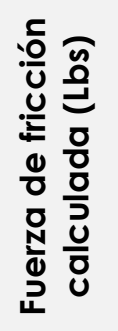 & 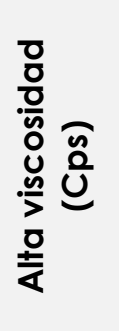 & 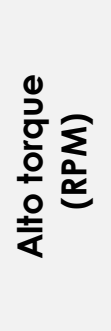 & $\begin{array}{l}\frac{a}{0} \\
0 \\
0 \\
0 y \\
0 \\
0 \\
0 \\
0 \\
0 \\
0\end{array}$ & 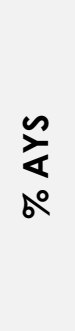 & 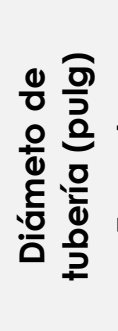 & 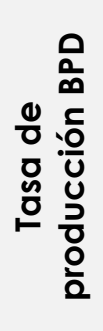 & 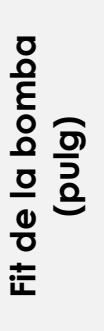 & 훙 \\
\hline $\begin{array}{l}\text { Alta viscosidad } \\
\text { (Cps) }\end{array}$ & 10 & 9 & 8 & 7 & 8 & 0 & 8 & 4 & 4 & 7 & 7 & 8 & 80 \\
\hline $\begin{array}{l}\text { Alta viscosidad } \\
\text { (Cps) }\end{array}$ & 9 & 10 & 8 & 8 & 8 & 8 & 0 & 4 & 6 & 6 & 7 & 1 & 75 \\
\hline $\begin{array}{l}\text { RGP } \\
\text { (pues } 3 / \text { bbls) }\end{array}$ & 4 & 4 & 4 & 2 & 4 & 4 & 4 & 0 & 2 & 1 & 3 & 1 & 33 \\
\hline$\%$ AYS & 6 & 8 & 5 & 3 & 5 & 4 & 6 & 2 & 0 & 1 & 2 & 1 & 44 \\
\hline $\begin{array}{l}\text { Diámeto de } \\
\text { tubería (pulg) }\end{array}$ & 6 & 6 & 6 & 6 & 6 & 7 & 6 & 6 & 1 & 0 & 1 & 1 & 47 \\
\hline $\begin{array}{l}\text { Tasa de } \\
\text { producción } \\
\text { BPD }\end{array}$ & 7 & 9 & 5 & 5 & 6 & 7 & 7 & 3 & 3 & 1 & 0 & 3 & 56 \\
\hline $\begin{array}{l}\text { Fit de la } \\
\text { bomba (pulg) }\end{array}$ & 8 & 6 & 7 & 7 & 6 & 8 & 1 & 1 & 1 & 1 & 3 & 0 & 49 \\
\hline Total & 83 & 76 & 71 & 64 & 70 & 80 & 75 & 33 & 44 & 47 & 56 & 49 & \\
\hline
\end{tabular}

Fuente: Elaboración propia

Como seguimiento de esta simulación de los pozos con el programa actividad, fue indispensable recolectar cierta información de cada pozo tales como: completación actual de los pozos, producción neta por día, FICCIION XLS.

pruebas asociadas a la producción, comportamiento de producción, configuración mecánica, datos del valor de separación elevador-grapa de la barra pulida del balancín (SENO), entre otros, para lograr realizar la

\section{RESULTADOS}

Para la validación de los resultados de la tecnología inductor térmico NOKALT 4500 se tomaron en cuenta los aspectos operacionales y de instalación, tal como se muestra en la Figura 2. 


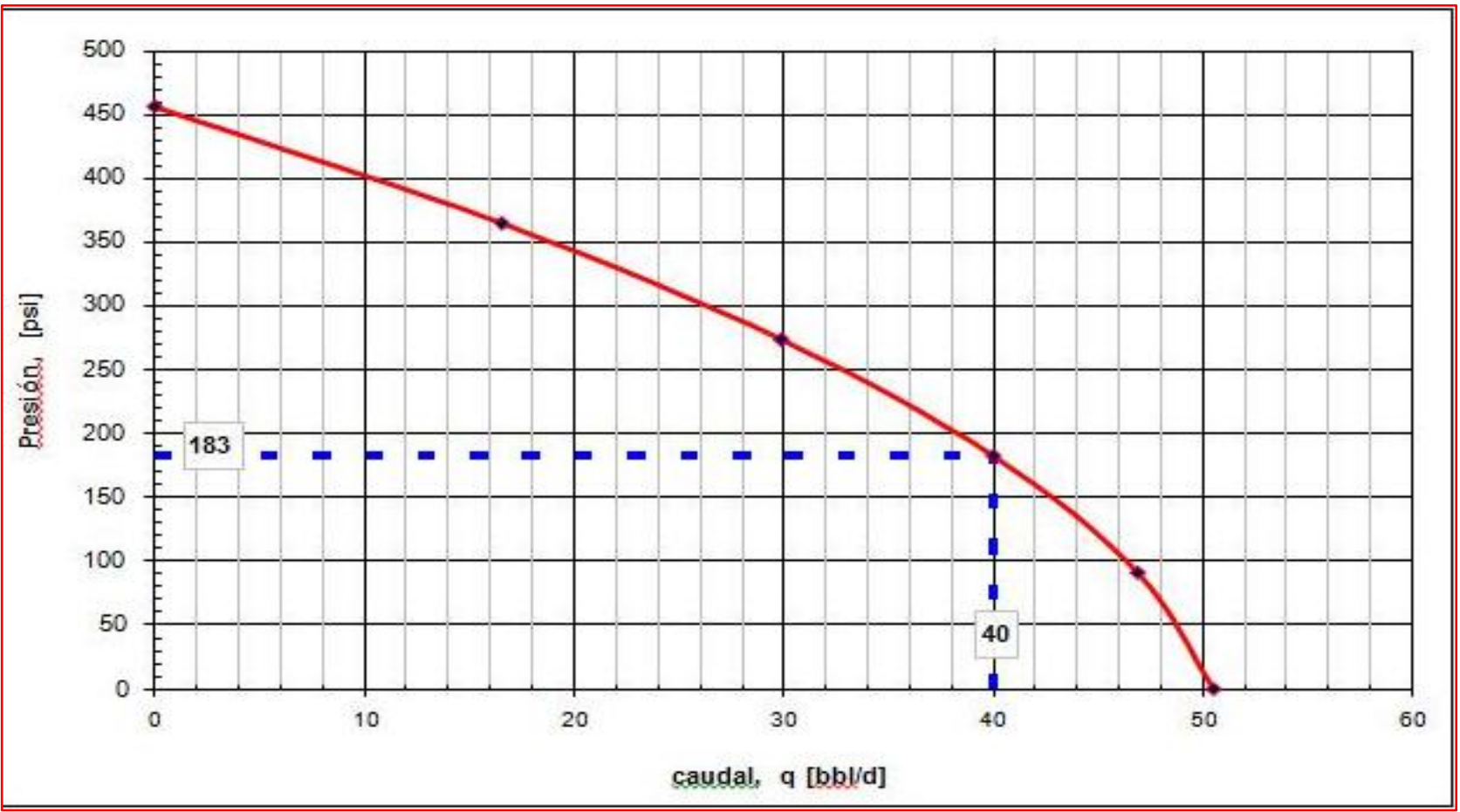

Figura 4. IPR del pozo con el inductor NOKALT 4500. Fuente: Elaboración propia

En la Figura 5, se observa la caracterización del crudo del pozo, donde se visualiza la reducción de la viscosidad, por el aumento de la temperatura del crudo.

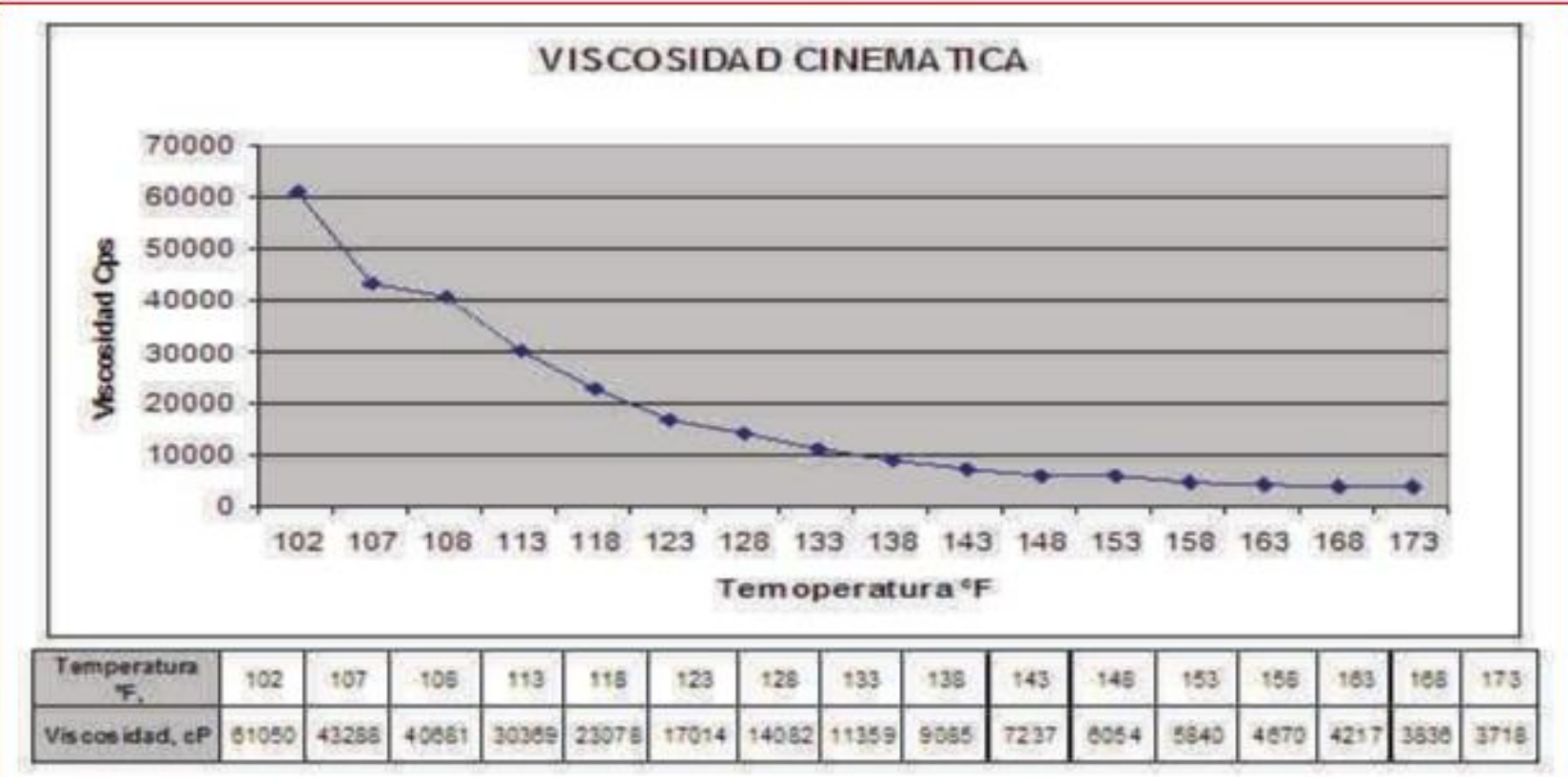

Figura 5. Caracterización del crudo viscosidad Vs temperatura del pozo. Fuente: Elaboración propia 
Según la corrida realizada, se observó que el pozo quedó optimizado con una producción promedio de 40 bnpd Vs una producción promedio de 21 bnpd que presentaba el pozo sin la instalación del sistema inductor térmico NOKALT 4500, obteniendo así un ganancial de 19 bnpd sobre su comportamiento estadístico, el cual representó un $95 \%$ de incremento de su tasa de producción.

Posteriormente se construyó las curvas de afluencia (Inflow performance relationship) para cada pozo seleccionado en condiciones de producción sin estimulación térmica (fluido frío), y con calentamiento eléctrico de fondo (fluido caliente). En la Figura 6, se muestra la curva de afluencia del pozo observándose que en la producción en frío el pozo puede alcanzar una tasa de producción de 36 BPD, cuyo caudal máximo (Qmax) es de 52 BPD, presentando una alta fricción cabilla-fluido por el orden de 6 pulg y con la tecnología inductor térmico NOKALT 4500, puede alcanzar una producción de 50 BBPD (variación +14BPD) eliminando la alta fricción cabilla-fluido, lo que incrementa la vida útil del equipo de bombeo de superficie y de subsuelo.

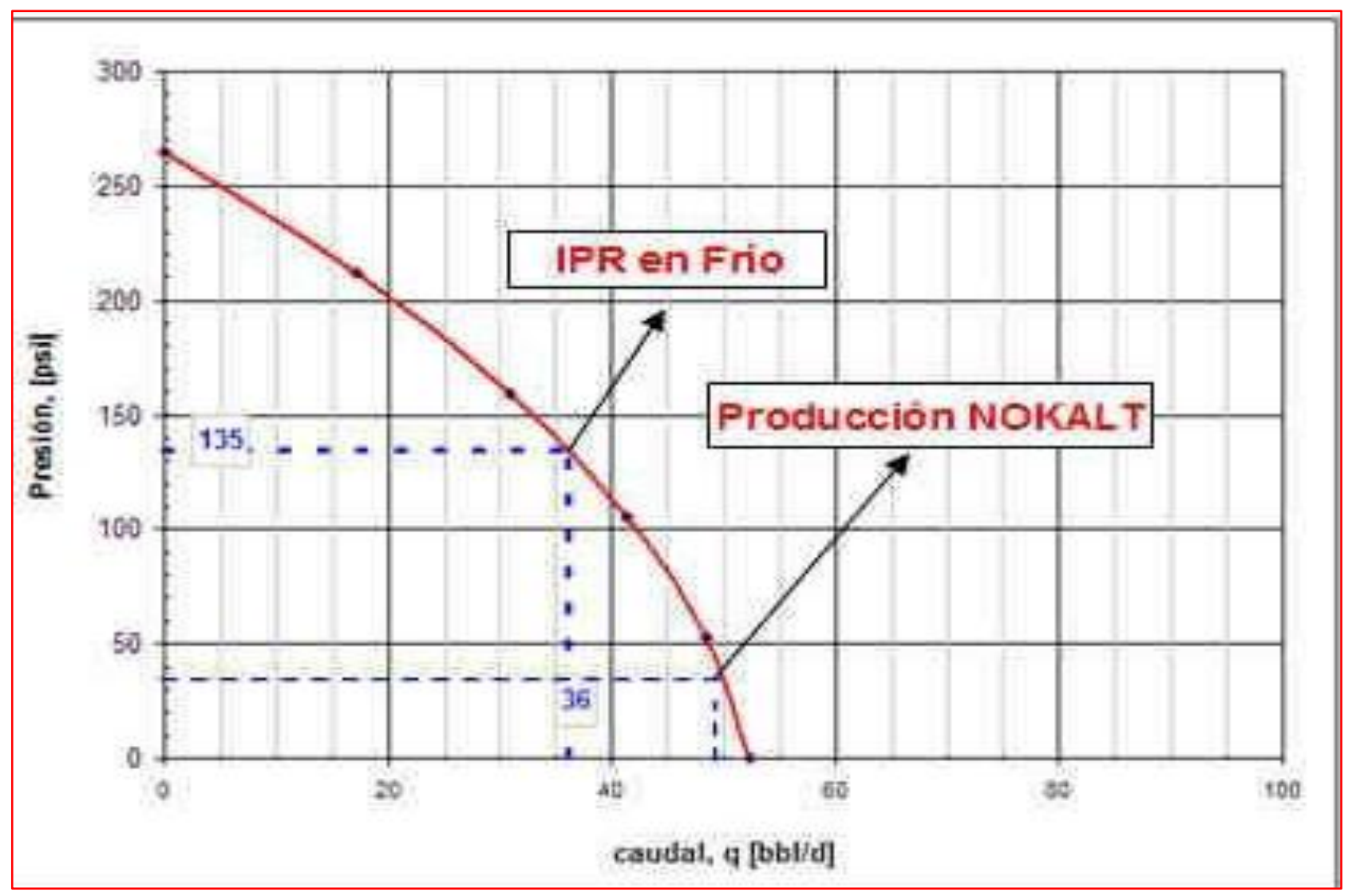

Figura 6. Gráfico de la curva de afluencia del pozo. Fuente: Elaboración propia 
Finalmente y a partir de estos resultados, se creó una nueva matriz de evaluación, para seleccionar aquellos pozos que presentaron las condiciones más idóneas para la instalación de la tecnologíainductor térmico NOKALT 4500, a los que se realizaron los cálculos de viscosidad (100, 150,180) º, a través de la ecuación de Beggs. Se evaluaron seis (6) pozos de bombeo mecánico, y se observó que con la utilización de la tecnología de calentamiento eléctrico en fondo NOKALT 4500, la producción pasaría de 264 BPD a 396 BPD, lo cual representa un ganancial de 132 BPD que equivale a un incremento de producción del 50\%.

De igual manera, se evaluaron cuatro (4) pozos seleccionados de Bombeo por Cavidad Progresiva, donde se observa que con la utilización de la tecnología de calentamiento eléctrico en fondo NOKALT 4500, la producción pasaría de 126 BPD a 232 BPD, lo que representa un ganancial de 106 BPD equivalente a un incremento de producción del $84 \%$.

\section{CONCLUSIONES}

Quedo demostrado que con la instalación del inductor térmico NOKALT 4500, y a través de ajustes periódicos de la velocidad de bombeo y el aumento de la carrera del balancín se logra mejorar la tasa producción promedio de 21 BNPD a 40 BNPD representando así un $95 \%$ de incremento en la tasa de producción.

Por otro lado, a través de la matriz escalonada, que comprende los siguientes criterios: seno, THP, cargas sobre la barra pulida, fuerza neta calculada, fuerza de fricción calculada, viscosidad, torque resistivo, RGP, \%AyS, diámetro de tubería, tasa de producción y fit de la bomba; se logró realizar una selección de los mejores pozos para poner en funcionamiento el inductor térmico NOKALT 4500.

\section{REFERENCIAS}

Hurtado, J. (2006) "El proyecto de investigación". 4ta Edición. Ediciones Quirón Sypal. Colombia

PDVSA (2015. Tecnologia No-kaltß). Disponible en: https://docplayer.es/95013742-

Tecnologia-no-kalt-servicios-deenergia.html

PDVSA. (2013) "Misión y Visión de Petróleos de Venezuela", información disponible en www.pdvsa.com, consultada el 7 de febrero de 2014

Salager JL, Antón RE., Sabatini DA, Harwell JH, Acosta E, Tolosa LI, (2005). Enhancing Solubilization in Microemul-sions - State of the Art and Current Trends". J SurfactantsDetergents, Vol. 8, pp. 321 